\title{
Pour une analyse et une pratique de l'environnement au-delà des secteurs
}

\author{
Entretien avec Bernard Kalaora
}

\author{
Bernard Kalaora et Arthur Laurent
}

Bernard Kalaora est socio-anthropologue, chercheur associé au laboratoire d'anthropologie des institutions et organisations sociales (EHESS/LAIOS) et professeur honoraire de sociologie de l'environnement à l'Université Jules Verne d'Amiens et est consultant auprès d'organisations nationales et internationales et président de l'association LITOCEAN. Ses domaines d'intérêt portent tout particulièrement sur la construction sociale de l'environnement et son inscription dans l'espace public dans une perspective associant les sciences sociales et les sciences de l'environnement.

Vos recherches ont porté sur des enjeux environnementaux qui dégagent clairement des dimensions transnationales. Pouvez-vous nous expliquer comment cette dimension vous est apparue et comment l'avez-vous traitée?

Cette dimension, je l'ai rencontrée sur le terrain dans mon activité d'expertise pour le compte de la Commission européenne sur des programmes de gestion intégrée des zones côtières. C'est au cours de cette pratique que j’ai été pris dans un système complexe avec une faible marge de manœuvre. À la suite de plusieurs terrains sur la méditerranée (Algérie, Égypte, Maroc, Tunisie), j’ai essayé de me poser pour prendre des distances. J'ai alors écrit l'article « Global Expert» dans la revue Ethnologie française en 1999 dans lequel j'ai tenté de montrer comment s'opère la mise sur orbite planétaire de l'expertise qui va au-delà d'une seule entreprise de normalisation, au sens ou en effet à travers l'expertise on reconfigure la carte et le territoire. On les déconstruit pour qu'ils répondent à des procédures d'évaluations externes, celles des institutions onusiennes et européennes. Les termes de référence propres à l'expertise présentent une série de définitions, de cadrages et des demandes de mise en place de groupes de travail et de synergie entre les groupes de terrain. Ce que je montrais dans cet article, c'est comment la globalisation au travers d'un formatage extérieur redéfinissait les cadres d'appréhension des problèmes au-delà des échelles nationales, ce qui représentait un processus de dénationalisation auquel bien souvent les populations locales opposaient une résistance. La convention de Barcelone ayant été définie sans concertation avec les différentes populations concernées, lesquelles se retrouvaient confrontées à une approche, la GIZC (la gestion intégrée des zones côtières) qui remettait en question leur façon de travailler, mais aussi leur représentation du territoire.

Ces processus standards d'une démocratie par les instruments (ceux de l'expertise et de la gouvernance) dans des régimes, y compris dictatoriaux ou militaires (Tunisie, Algérie, Égypte) se traduisaient sur le plan de la sémantique de l'expert par le parler neutre, c'est-à-dire un langage dont la valeur émotionnelle est faible contrairement à 
sa valeur instrumentale : gestion, management, capacity building, gouvernance et participation, mots qui, sciemment, évitent toute allusion idéologique ou politique et qui fait du mode managérial un modèle à imiter quel que soit les contextes. J'avais été très influencé par l'ouvrage Imperial Nature de Goldman (2005) qui montrait que la question de la nature et de l'environnement pouvait être un instrument de recomposition et de redéfinition des modalités de pouvoir, cet auteur voyant l'emprise de la question de la nature comme une nouvelle forme d'impérialisme plus fluide, en termes d'équipement de pouvoir, mais aussi en termes de cadrage des problèmes et de requalification des territoires. Ensuite j'ai aussi été influencé par l'ouvrage de Saskia Sassen La globalisation, une sociologie (2007) qui montrait comment cette globalisation était aussi un processus complexe qui peut être activé au sein même de l'État national. Étudier le global implique de se concentrer non seulement sur ce qui en échelle est explicitement global, mais aussi de voir comment ces processus globaux initiés par des organisations internationales pénètrent et transforment les pratiques à l'échelle locale. Ces approches théoriques recoupaient ce que j'avais vécu sur le terrain en montrant comment les experts internationaux font totalement abstraction de la donne locale dans des projets qui se font dans une échelle de temps courte et qui produisent des « désassemblages du territoire » et du droit auprès des pays qui font l'objet de l'expertise globale. L'expert ne recourt pas à des sources locales et concrètes pour son travail, il a pour référence uniquement la littérature produite par les experts internationaux et cette littérature est complètement extérieure aux dimensions locales. La globalisation apparaît comme une nouvelle forme d'universalisme.

Bien que dans mes travaux je n'utilise pas forcément ce terme de transnational, je me sens proche de cette réflexion et cela au même titre que Sassen qui montre comment la globalisation impacte les relations entre les États nations et produit du global dans le local. La globalisation implique deux dynamiques, la première induit la formation d'institutions, de processus et d'instrument explicitement globaux, comme la banque mondiale, l'Union européenne, les marchés financiers, les ONG, etc. La deuxième dynamique œuvre à une autre échelle. Des réseaux inter-frontaliers d'acteurs s'engagent dans des activités locales avec un objectif global comme c'est souvent le cas pour les questions d'environnement. On peut dans ce cas de figure parler de transnational. Dans cette perspective Sassen montre également que l'on assiste à de nouvelles formes de mobilisation qui recoupent clairement des sujets environnements transnationaux tels les enjeux de biodiversité, désertification, bio invasion, etc. Cela me semble important de souligner que ces mobilisations sont mobiles, pas nécessairement pérennes avec des gens qui se rencontrent à l'occasion de différents sujets. D'où la difficulté pour les sociologues d'étudier ces formes hybrides et aussi présentes dans différents lieux.

Dans ce cadre, comment les enjeux environnementaux modifient-ils selon vous l'exercice du pouvoir?

En fait, on a là une des formes de l'exercice du pouvoir qui dépasse les figures classiques et qui tient au fait que les questions d'environnement ne répondent pas au formatage classique des appareils institutionnels. Ce sont le plus souvent les acteurs concernés 
par ces enjeux qui enquêtent et définissent les problèmes (au sens de Dewey 2010) que devront prendre en charge les décideurs. En découle pour l'État une question sur l'identification de ces acteurs ainsi que sur la hiérarchisation des problèmes à traiter. L'État se retrouve confronté à une multiplicité d'acteurs qu'il doit reconnaître et qui reconfigurent l'exercice normal du pouvoir.

Mais un autre aspect de modification de cet exercice du pouvoir est lié à une approche historiquement sectorielle de l'organisation alors que le monde environnemental est profondément transnational. Ainsi la constante redéfinition en France du ministère de l'environnement depuis son existence en 1971 en est un symptôme. En fait, selon les sujets, ce n'est pas un secteur ministériel qui peut traiter le sujet, mais une multiplicité de secteurs avec lesquels il faut composer et négocier. On est donc constamment confrontés à une difficulté, liée sans doute à une dimension pyramidale du pouvoir en France, qui consiste à constamment rajouter des couches plutôt qu'à redéfinir les problèmes. L'environnement c'est un enjeu de technocratie fort, car les grands corps de l'État fortement structurés peinent à être redéfinis tels par exemple les corps des mines ou celui des eaux et forêt, etc. On assiste à une certaine inertie et une multiplication des approches chacun se sentant propriétaire de son domaine de compétence, par exemple les mines disant l'environnement c'est nous ou l'environnement ce sont les risques et le Corps des Forêts disant l'environnement c'est la nature et c'est nous. J'ai pu voir cela au sein du ministère entre la gestion de la nature et des risques où l'on aboutit à des formes d'incompatibilité totale. Là où les corps des mines travaillent de front avec les industriels et où le risque se négocie, dans l'approche de la protection de la nature on vise d'abord à protéger et à sanctuariser la nature et à considérer les activités industrielles et marchandes comme des ennemis. C'est donc une approche totalement différente. Cette contradiction se voit au quotidien dans la gestion du ministère (entre les différentes directions) avec des effets pervers en termes de hiérarchie et de légitimité. Ainsi, là où l'environnement demande une adaptation et une souplesse permanente, en France l'approche très rigide et sectorisée rend impossible une approche adaptative en fonction des situations et des contextes. L'environnement exige des approches pragmatiques, de connaissance, de gestion et de gouvernance même si je n'aime pas ce terme.

Justement, la notion de gouvernance a notamment été appliquée aux enjeux environnementaux, vous semble-t-elle effectivement opératoire dans les cas que vous avez pu étudier?

La gouvernance me semble tout à fait opératoire en termes de récit et de discours, car ce terme comme le développement durable a entrainé dans son sillage les gouvernements à travers les vecteurs que sont les institutions onusiennes porteuses de ce langage. Il y a donc une efficacité assez remarquable. Ainsi il y a des outils en très grand nombre dans lesquels se reconnaissent un milieu, une communauté épistémique et de nouvelles couches sociales. De ce point de vue il y a une grande efficacité du terme de gouvernance.

Maintenant, est-ce opératoire sur le plan d'une meilleure efficacité de la gestion des enjeux environnementaux ? Rien n'est moins sûr, car la gouvernance a à voir avec 
l'aplatissement de problèmes. On assiste à une sorte de réductionnisme, d'horizontalité. On le voit bien dans le livre d'Aykut et Dahan (2015) qui d'ailleurs a un point d'interrogation (NDLR. «Gouverner le climat?»), on voit que c'est un leurre. Lorsque l'on reste à un niveau d'abstraction des controverses avec d'un côté la science et d'un côté le politique, on arrive certes à un certain niveau de production de connaissance, mais compte tenu de l'échelle de traitement des problèmes, il n'y pas de prise concrète au niveau des politiques et en particulier à celui plus fin des élus et des collectivités locales qui ne savent pas comment et par quel bout traiter de la question. On voit un manque d'articulation entre les échelles globales locales et régionales. La question du climat, la COP 21 l'a bien montré, doit se traiter à l'échelle de territoires et de pays singuliers et non à celle globale de la planète.

La gouvernance pour moi doit être remise en contexte dans le processus de la «globalisation heureuse " puis de l'idée que nous sommes tous en danger et qu'il y aurait des solutions que tous devraient appliquer avec un pari un peu fou d'une commune mesure de l'humanité. On voit aujourd'hui les réactions et potentiels effets pervers de cette illusion de l'homogénéisation des parcours, incompréhensions replis identitaires, sur la localité, etc. Cela montre bien que derrière cette idée de gouvernance se cache une idée de néolibéralisme et d'une définition du monde par l'économie et le marché et qu'on passe à côté de la question de "gouvernementalité » et de la souveraineté. Il n'est pas certain que cela perdure. Padioleau, proche de la sociologie de l'organisation de Crozier, évoquait de manière méprisante dans les années 2000 «les gogos de la gouvernance», un mouvement selon lui néo conservateur visant à faire du marché l'instrument de régulation des sociétés et prônant la fin des séparations entre public, privé et société civile. La gouvernance dans cette perspective ne serait que la loi du plus fort au mépris du droit et de l'intérêt général, ciment de la république et du lien social.

Quelles sont selon vous les grandes caractéristiques des enjeux environnementaux au niveau global actuellement?

On voit un rabattement sur la notion climatique et une résurgence de la question de la pollution de l'air liée aux problèmes de santé qui sont mis plus en avant aujourd'hui alors que les questions de bruit présentes dès la fondation du ministère au sein d'une Délégation du cadre de vie ont disparu de l'agenda gouvernemental ou du moins sont secondaires alors que le bruit reste l'une des premières préoccupations des Français. La quotidienneté et le bien-être sont plutôt en retrait il me semble, il faudrait faire des recherches sur cela pour voir comment les problèmes sont hiérarchisés. Il me semble que l'on met de nos jours beaucoup plus en avant les questions les plus globales avec le climat et la pollution de l'air. La biodiversité qui avait du mal à émerger devient elle aussi importante avec le «GIECC biodiversité ». Cela reste traité toutefois à des échelles globales. Aussi, les enjeux de bio-invasion sont importants, mais peu visibles, ils concernent les spécialistes de la conservation et de la protection. La question des océans émerge comme un enjeu global, mais il est d'autant plus difficile à intégrer dans les politiques publiques qu'il est hors sol (invisible aux yeux des terriens à l'exception du 
rivage) et que les liens entre terre et mer ne sont absolument pas faits dans les imaginaires collectifs. L'océan s'arrête au rivage à la plage ou au maximum aux 20 miles du territoire maritime et échappe aux politiques publiques. La gestion intégrée des zones côtières a beaucoup de mal à intégrer tout ce qui vient de la mer. Ainsi au lieu de GIZC (Gestion intégrée des zones côtières) on parle GILM (Gestion intégrée mer et littoral) pour montrer le lien et le besoin de faire le passage entre les deux. Ce lien toutefois n'est pas relayé au niveau institutionnel. Par exemple le Conservatoire du littoral s'occupe essentiellement du domaine terrestre et côtier et l'Ifremer de la mer et la coopération entre ces deux institutions se réduit au minimum même si chacune prétend faire de la gestion intégrée...

Il y a quelques années vous avez coécrit, avec Chloé Vlassopoulos, un livre intitulé Pour une sociologie de l'environnement (2010). Comment avez-vous traité dans ce cas la dimension transnationale?

Pour une sociologie de l'environnement est un livre épistémologique qui vise à questionner la façon dont en France les sciences sociales ont investi ce champ qui lui était totalement inhabituel. Nous essayons de montrer que cette question ne peut pas être la propriété d'une discipline. Je ne sais pas si c'est pour autant de la pluridisciplinarité, je préfère parler d'interaction et de coopération entre disciplines. L'environnement ce sont tout à la fois des processus naturels sociaux, écologiques, politiques et il est totalement illusoire de penser que l'on peut segmenter ces problèmes et les traiter de manière catégorielle, de façon disciplinaire. Cela suppose des formes de coopération. Ainsi quand on se donne un objectif de langage commun comme ce fut le cas avec le Groupe de sociologie rurale du CNRS dont faisait partie Marcel Jolivet, on voit vite les limites de l'exercice. Il y a cependant des formes de collaboration possible si la question est soulevée pars des acteurs publics ou sociaux. C'est dans ce cadre qu'on peut faire collaborer et évoluer les disciplines, j'ai pu expérimenter cela au sujet d'un problème de bioinvasion dans une forêt proche d'Orléans avec G. Decocq (pharmacologue) et A. Javelle (ethnologue). Nous avions mis en place un dispositif regroupant différentes disciplines, géographes, mathématiciens, juristes, ethnologues, politologues en y incluant les gestionnaires forestiers et les associations de protection concernés au premier chef par les bio-invasions. Les problèmes se redéfinissaient dans ces interactions des uns avec les autres, les géographes comme les mathématiciens ou les politologues à l'issue des questions posées par les gestionnaires et milieux associatifs. On se trouvait alors dans un processus de construction, de recherche qui n'était jamais stabilisée. On ne cherchait pas un langage commun, mais une réflexivité.

Je reviens au livre Pour une sociologie de l'environnement dont l'objectif principal était de faire un diagnostic sur les manières donc les sciences sociales ont abordé le problème depuis la création du ministère de l'environnement en 1971 jusqu'à nos jours. L'idée était de donner des pistes pour travailler sur des problèmes non stabilisés et marqués par l'incertitude alors que les sociologues avaient l'habitude de traiter de sujets très stabilisés et de chercher à échapper au péril de l'incertitude. Et aussi au-delà de constituer une science sociale en phase avec la globalisation et non plus centrée sur 
l'État nation. C'est dans ce cadre que la sociologie académique s'était construite en même temps que l'État-nation et y était donc très attachée. Cela supposait donc aussi de travailler avec le monde anglo-saxon, car c'est là que ces démarches sont sans doute bien d'avant-garde et plus avancées surtout du point de vue des sciences sociales. Là, la bibliographie est immense, beaucoup plus qu'en France. En France on a du mal à mutualiser ces travaux, car on manque non pas d'un langage commun, mais d'un milieu qui reste assez éclaté et fragmenté. De ce point de vue nous sommes très différents des Américains ou même des Allemands qui ont réussi à créer des instituts sur l'environnement. On a eu cette volonté en France à un moment, mais elle n'a pas abouti (Humanités environnementales 2017).

Plus récemment, avec Bernard Decocq et Chloé Vlassopoulos (2016), vous avez proposé un nouveau regard sur le rôle de la forêt dans la gouvernance mondiale du climat. Quel rôle la forêt joue-t-elle dans le contexte actuel ?

Cette réflexion est à remettre dans son contexte d'une série de travaux antérieurs avec Antoine Savoye, dont La forêt pacifiée (1986). Nous avions étudié les forestiers de montagne influencés par l'école de Le Play et la méthode monographique. Ils produisaient une vision de forêt et de développement ne séparant pas la mission forestière de celle de développement des régions de montagne. Ils avaient une réflexion sociale sur les modes de vie pastoraux et leur objectif n'était donc pas la «nationalisation du sol» et l'expulsion des populations, mais au contraire une vision intégrée. Il y avait aussi une approche de rationalisation de modes pastoraux pour en faire une économie marchande. Ils étaient très peu nombreux, une vingtaine entre 1860 et 1914, mais avec une vraie vision sociale. La forêt salvatrice c'est donc l'autre pendant, le versant de l'administration centrale, celui d'une vision nationale des forêts de montagne comme forêt régénératrice au sens fort du terme, de la nation et de l'identité française. Ce discours s'est construit au travers de la question des inondations sous le Second Empire et sur l'idée que la forêt permettait de résoudre les problèmes sociaux comme la santé et de l'hygiène (tels par exemple de la lutte contre la malaria et le choléra). On a même argumenté sur le rôle patriotique de la forêt pour lutter contre le déboisement, car un peuple sans forêt ce sont des corps amoindris et des populations affaiblies. Les Prussiens ayant de belles forêts, leur population est plus vigoureuse et ce sont les différences entre les taux de boisement de ces deux pays qui seraient la cause de la défaite militaire des Français. Ces forestiers ont aussi puisé des arguments dans les sciences (météorologie, hygiénisme, économie, hydrologie, etc.) pour faire valoir des intérêts corporatistes. Il y a là un positionnement face aux acteurs de Ponts et Chaussées, car il s'agit de faire valoir la légitimité du corps des forestiers par rapport à la question des inondations et du reboisement des montagnes. Ainsi le corps forestier affaibli depuis la Révolution française devient l'élément majeur d'une production de l'espace et de sa requalification moderne. Les forêts sont les piliers de l'aménagement du territoire. Un débat public avec une stratégie de communication efficace va convaincre les autorités publiques à la nécessité du reboisement des montagnes et à l'éviction des populations 
locales (les pasteurs) dont les usages archaïques sont contraires à la modernisation du territoire et au désenclavement des régions de montagne.

L'argumentation environnementale avant d'être un facteur de conservation des ressources naturelles est dans ce contexte un vecteur de modernisation de la France. On voit un rapport avec ce qui se passe aujourd'hui avec la forêt carbone. Chloé Vlassopoulos montre comment le mécanisme REDD, mécanisme abstrait et déterritorialisé avec des procédures très bureaucratiques exerce une certaine violence sur les populations locales qui se traduisent, comme le montre Sassen dans son dernier ouvrage Expulsions (2016), par des expropriations au détriment des populations locales. S’il est vrai que les forêts sont des puits de carbone, on voit que ce mécanisme induit non seulement des expulsions, mais aussi, comme le montre Guillaume Decoq des formes de culture sylvicole dynamique : pour stocker le carbone il faut avoir des espèces qui poussent vite et arrivent à maturité à moins 30 ans et là, de nouveau, l'arbre cache la forêt, la biodiversité est oubliée.

Quelles sont selon vous actuellement les grandes priorités de recherche sur la question des enjeux environnementaux transnationaux?

Prenons Angus Deaton, le prix Nobel d'économie. Dans son ouvrage La grande évasion » (2016), il aborde en tant qu'économiste la question des inégalités. Il montre que si l'écart entre pays se réduit, il faut aussi se poser la question de comment les écarts se creusent à l'intérieur des pays et l'on voit qu'ils sont en extension rapide. Cela me semble très important par rapport à cette question d'environnement. On a une tendance à traiter le problème de manière transnationale et globale et alors à avoir beaucoup de difficulté à identifier quelles sont les populations les plus impactées à différentes échelles du local au global. C'est effectivement très difficile, car ce n'est pas forcément les plus pauvres, cela peut être aussi les riches. En effet, la distribution est très aléatoire même s'il y a de très fortes chances que les plus pauvres soient plus impactés que les plus riches. Il me semble que l'on manque beaucoup de réflexion sur les facteurs spécifiques favorisant les inégalités environnementales. Il y a très peu de recherches en France alors que la question est plus développée dans le monde anglo-saxon avec la tradition de recherche sur la justice environnementale et les minorités ethniques.

Peu de travaux sur ce sujet en France pourtant il y a aussi des minorités, mais c'est un thème difficile à aborder et un travail donc à développer. Il me semble que la question des territoires périphériques n'est pas encore abordée avec une approche d'inégalités environnementales tant à une échelle globale qu'a une échelle locale en lien avec les inégalités concernant la santé et l'accès aux soins. Je vois aussi un champ de recherche sur les questions de genre très peu développé en France (le genre et l'environnement), voilà les deux priorités de recherche pour moi.

\section{Bibliographie}

AYKUT S., DAHAN A. (2015), Gouverner le climat? 20 ans de négociations internationales, Paris, Presses de sciences politiques. 
Blanc G., Demeulenaere E., Feuerhahn W. (dir.) (2017), Humanités environnementales. Enquêtes et contre-enquêtes, Paris, Publications de la Sorbonne.

Deaton A. (2016), La grande invasion. Santé, richesse et origine des inégalités, Paris PUF.

DecocQ G, Kalaora B., Valassopoulos C. (2016), La forêt salvatrice, reboisement, société et catastrophe au prisme de l'histoire, Paris, Champ Vallon.

DEWEY J. (2010), Le public et ses problèmes, Paris, Gallimard.

Javelle A., Kalaora B., DecocQ G. (2010), « De la validité d'une invasion biologique », Études Rurales, Proliférantes natures, vol. 185, p. 39-50.

Goldman M. (2005), Imperial Nature, New Haven, Yale University Press.

KalaORA B., SAVoye A. (1986), La forêt pacifiée. Les forestiers de l'Ecole de Le Play, experts des sociétés pastorales, Paris, L'Harmattan.

Kalaora B. Valassopoulos C. (2013), Pour une sociologie de l'environnement: environnement, société, politique, Paris, Champ Vallon.

KALAORA B. (1999), «Global expert, la religion des mots », Ethnologie Française, vol. 29, $\mathrm{n}^{\circ}$ 4, p. 513-527.

PADIOLEAU J.C. (2000), « La gouvernance ou comment s'en débarrasser, les gogos de la gouvernance», Libération du 1 juin 2000.

SASSEN S. (2007), La globalisation. Une sociologie, Paris, Gallimard.

SASSEN S. (2016), Expulsions. Brutalité et complexité dans l'économie globale, Paris, Gallimard. 